\title{
Per-band Link Control Transients Protection in Distributed Fiber Raman Amplifier Cascades
}

\author{
Victor Pincheira, Marcelo A. Soto and Ricardo Olivares \\ Electronic Engineering Department, Universidad Técnica Federico Santa María, Av. España 1680, Casilla 110-V, Valparaíso, Chile, rov@elo.utfsm.cl
}

\begin{abstract}
In this work we introduce the use of the per-band link control method in cascades of distributed fiber Raman amplifiers (DFRAs) for compensating power transients of WDM channels. By using two compensation channels, each one controlled by a proportional-integrative electronic control circuit and assigned to a particular signal band, the power excursion achieved in the surviving channels, in a cascade of 10 DFRAs, is reduced from $4.88 \mathrm{~dB}$ to $0.24 \mathrm{~dB}$, when 17 out of 20 channels are added or dropped. By adding more compensation channels, this transient protection technique can be extended to wideband DFRAs applications.
\end{abstract}

Keywords-Distributed Raman amplifiers, link control protection.

\section{INTRODUCTION}

Distributed Fiber Raman Amplifiers (DFRAs) have become innovative photonic devices that have allowed to exploit the potential of wavelength division multiplexed (WDM) optical systems. These amplifiers may provide bandwidths as large as $12 \mathrm{THz}$, flexible gain and bandwidth design, low noise, and great capacity to mitigate fiber nonlinearities effects. These characteristics make DFRAs advantageous optical components compared with traditional lumped fiber amplifiers such as Erbium-Doped Fiber Amplifiers (EDFAs) [1]. However, the use of DFRAs in Wavelength-Routed Optical Networks (WRONs) has certain impairments, similar to those present when using EDFAs, since the optical power into the network continuously changes. WRONs may actually present a variable number of channels, due to a dynamic reconfiguration of the network (e.g. for fault correction), channel addition/removal at nodes, or due to increasing network capacity [2]. Depending on the situation, the number of channels may go up or down, giving rise to power transients at the output of the DRFAs, representing a major limitation to the performance of WDM networks. If some channels are dropped, the power in the surviving channels may surpass the threshold above where the fiber nonlinearities can no longer be neglected. If channels are added, the power in the surviving ones diminishes, and may fall below the receiver sensitivity. In order to maintain the overall network performance, the power of the surviving channels must be kept within acceptable limits. For that, several transient control techniques have been proposed, of which the main three are referred to as pump control, link control, and laser control [3]-[9]. One of them, the link control protection technique [4], is preferred for its simplicity and efficiency. In this work we investigate the application of this technique, but over the basis of segmented bandwidth, referred to here as per-band link control protection method, for suppressing power transients in cascades of wideband DFRAs.

This work is organized as follow. Section II describes the general aspects associated to the link control technique applied to a cascade of DFRAs. Section III presents the mathematical model of the DFRA. Sections IV and V show simulation results after applying the link control protection technique to a cascade of 10 DFRAs. Section VI introduces the per-band link control scheme and its application to transient suppression in a wideband DFRA cascade. Finally, conclusions are presented in Section VII.

\section{Link Control Protection TeChNiQue}

The link control technique was originally developed for the transient control in cascades of EDFA and presented for first time by Zyskind et al in 1996 [4]. It is worth mentioning that the word "link" refers to a network segment between two nodes, where channels can be added or dropped.

When the link control technique is applied to a cascade of DFRAs, a control signal, with wavelength $\lambda_{c}$, is inserted before the first amplifier of the link, by using a wavelength select coupler $\left(\mathrm{WSC}_{1}\right)$, and is dropped before the last amplifier of the link $\left(\mathrm{WSC}_{2}\right)$ to avoid unnecessary load on the network. The power of the control signal is continuously adjusted by using an electronic feedback circuit, so that the total power at the input of the first amplifier is kept constant. Hence, the load on all amplifiers of the link is also constant, avoiding power transients. The control circuit is a proportional-integrative electronic control, whose mathematical representation is [10]:

$$
\tau_{i} \frac{d P_{c c}(z=0, t)}{d t}=k_{p} R(t)+k_{p} \tau_{i} \frac{d R(t)}{d t}
$$

where $P_{c c}(z=0, t)$ is the power of the control channel at the input of the first amplifier as a function of time. The parameters $k_{p}$ and $\tau_{i}$ represent the gain of the proportional and integrative error, respectively, which are used in the feedback loop. The error function $R(t)$ is defined as [10] :

$$
R(t)=\left[P_{t o t}(z=0, t=0)-P_{t o t}(z=0, t)\right]
$$

where $P_{t o t}(z=0, t)=\sum_{k=1, N} P_{k}(z=0, t)$ is the total power, including signal and control channel, at the input of the amplifier. The values of the parameters $k_{p}$ and $\tau_{i}$ are determined based on the dynamic of the feedback loop. Considering a control circuit response time of $4 \mu$ s (typically associated to real electronic components), the values of these parameters are $k_{p}=100$ and $\tau_{i}=10^{-6}$. 


$$
\begin{aligned}
& \frac{\partial P^{ \pm}(z, t, v)}{\partial z} \pm \frac{1}{V_{g}(v)} \frac{\partial P^{ \pm}(z, t, v)}{\partial t}=\mp \alpha(v) P^{ \pm}(z, t, v) \pm \gamma(v) P^{\mp}(z, t, v) \pm P^{ \pm}(z, t, v) \cdot \sum_{\varepsilon>v} \frac{g_{R}(v-\varepsilon)}{K_{e f f} \cdot A_{e f f}} \cdot\left[P^{ \pm}(z, t, \varepsilon)+P^{\mp}(z, t, \varepsilon)\right] \\
& \pm 2 h v \cdot \sum_{\varepsilon>v} \frac{g_{R}(v-\varepsilon)}{A_{e f f}} \cdot\left[P^{ \pm}(z, t, \varepsilon)+P^{\mp}(z, t, \varepsilon)\right] \cdot\left[1+\frac{1}{e^{h|\varepsilon-v| / K T}-1}\right] \Delta v \mp 4 h v \cdot P^{ \pm}(z, t, v) \cdot \sum_{\varepsilon>v} \frac{g_{R}(v-\varepsilon)}{A_{e f f}} \cdot\left[\frac{1}{e^{h|\varepsilon-v| / K T}-1}\right] \Delta v \\
& \mp P^{ \pm}(z, t, v) \cdot \sum_{\varepsilon<v} \frac{v}{\varepsilon} \frac{g_{R}(v-\varepsilon)}{K_{e f f} \cdot A_{e f f}} \cdot\left[P^{ \pm}(z, t, \varepsilon)+P^{\mp}(z, t, \varepsilon)\right] \mp 4 h v \cdot P^{ \pm}(z, t, v) \cdot \sum_{\varepsilon<v} \frac{g_{R}(v-\varepsilon)}{A_{e f f}} \cdot\left[1+\frac{1}{e^{h|\varepsilon-v| / K T}-1}\right] \Delta v \\
& \pm 2 h v \cdot \sum_{\varepsilon<v} \frac{g_{R}(v-\varepsilon)}{A_{e f f}} \cdot\left[P^{ \pm}(z, t, \varepsilon)+P^{\mp}(z, t, \varepsilon)\right] \cdot\left[\frac{1}{e^{h|\varepsilon-v| / K T}-1}\right] \Delta v
\end{aligned}
$$

\section{THEORETICAL MODEL}

In order to evaluate the performance of the proposed method when controlling transients in DFRAs, we have used the dynamic model of DFRA derived in [10] for our simulations. This model takes into account the generation and propagation of spontaneous emitted noise, generated by spontaneous Raman scattering process. In this work, we analyze WDM systems with a channel spacing separation of less than $1 \mathrm{~nm}$, so both spontaneous Stokes and anti-Stokes need to be undertaken [11]. This is because the gain/depletion caused by the spontaneous anti-Stokes process cannot be neglected in this case when being compared to the energy transfer produced by spontaneous Stokes Raman scattering.

Thus, when the attenuation of the fiber, Rayleigh backscattering, stimulated Raman scattering, and spontaneous Stokes and anti-Stokes scattering are considered, the propagation equation for co- and counter-propagating pumps, WDM channels, and spectral components of amplified spontaneous emission (ASE) powers, $P^{+}(z, t, v), P^{-}(z, t, v)$, describing their evolution in space and time, takes the form shown in equation (3). In this equation, $v_{g}(v)$ and $\alpha(v)$ are the group velocity and fiber loss coefficient at the frequency $v$, respectively, $\gamma(v)$ is the Rayleigh backscattering coefficient; $g_{R}(v-\varepsilon)$ is the Raman gain coefficient for an optical frequency

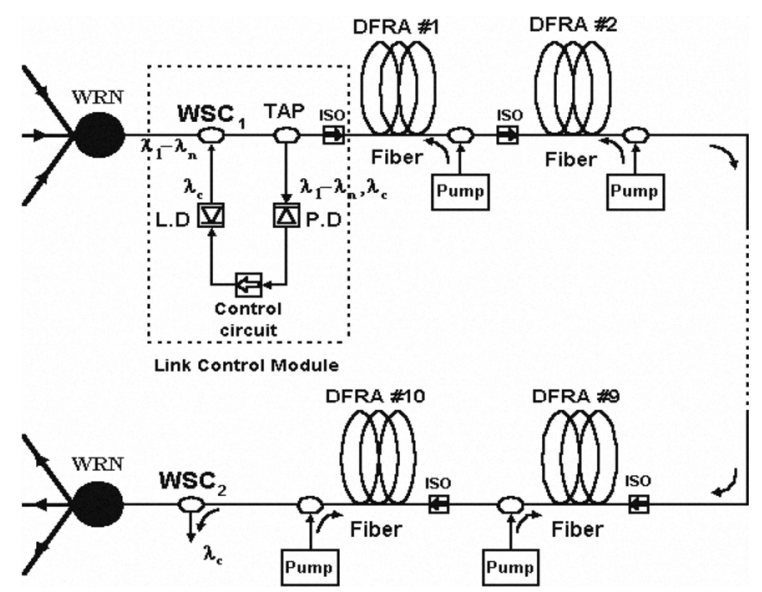

Figure 1. Schematic representation of the 10 DFRAs cascade incorporating link control protection. WRN: wavelength routed node; WSC: wavelength select coupler; LD: laser diode; PD: photo detector; ISO: isolator; $\lambda_{1}-\lambda_{n}$ : WDM channels wavelength, $\lambda_{\mathrm{c}}$ : compensation channel wavelength. difference $(v-\varepsilon) ; K_{e f f}$ is the polarization factor between pumps and Stokes signals; $A_{\text {eff }}$ is the effective interaction area of the fiber; $h$ is the Planck constant; $k$ is the Boltzmann constant; and $\mathrm{T}$ the absolute temperature of the fiber.

In order to obtain the evolution of the optical waves propagating along the fiber, in both space and in time domain, we solve equation (3) using a finite difference method, where discrete space and time domain elements are used in a grid of $M x N$ bins, with width $\Delta \mathrm{z}$ and $\Delta \mathrm{t}$, respectively. The equations are iteratively integrated for $\mathrm{z}=\mathrm{m} \Delta \mathrm{z}(\mathrm{m}=1, \ldots, \mathrm{M})$, at each time $\mathrm{t}=\mathrm{n} \Delta \mathrm{t}(\mathrm{n}=1, \ldots, \mathrm{N})$. For each space $(\mathrm{m} \Delta \mathrm{z} \rightarrow(\mathrm{m}+1) \Delta \mathrm{z})$ and time $(n \Delta t \rightarrow(n+1) \Delta t)$ increment, the corresponding variables are assigned values using their first order derivates. Note that the analysis carried out in this work focuses on systems based on cascades of DFRAs, so at each amplification stage the response of the previous stage is used as the initial condition for the next stage in the cascade.

\section{NUMERICAL SIMULATIONS}

Fig. 1 shows a schematic diagram of a transmission system between two wavelength-routed nodes (WRNs), which includes a cascade of 10 counter-propagating DFRAs with link control protection before the first amplifier. Each amplification stage is implemented by using $40 \mathrm{~km}$ of standard single mode fiber; with fiber attenuation coefficient of $0.19 \mathrm{~dB} / \mathrm{km}$ at the channel wavelengths and $0.23 \mathrm{~dB} / \mathrm{km}$ at the pump wavelengths, a peak Raman gain value $\left(g_{R}(\nu-\varepsilon) / A_{\text {eff }}\right)$ of $0.76 \cdot 10^{-3} \mathrm{~m}^{-1} \mathrm{~W}^{-1}$, and a wavelength-dependent Rayleigh scattering coefficient $\gamma(\lambda)=2.3510 \times 10^{-25} / \lambda^{3}[12]$.

In order to control the power transient of the channels when increasing/decreasing the total power at the input of the cascade, a compensation channel is added before the first DFRA in the link, and dropped after the last DFRA, by using wavelength selected couplers (WSC), as shown in Fig. 1. A portion of the total power of the WDM channels is detected at the input of the first DFRA, in order to have a feedback for the link control module. Transient control is carried out by automatically adjusting the power of the compensation channel, by applying a fast proportional-integrative electronic control circuit (PI controller), aiming at maintaining a constant total input power into the first DFRA. Thus, the channel loading is kept constant for all the DFRAs in the link.

In this work we have considered the propagation of 20 WDM channels at wavelengths ranging from $1545 \mathrm{~nm}$ (Ch. 1) 
to $1560.2 \mathrm{~nm}$ (Ch. 20), with $0.8 \mathrm{~nm}$ spacing and input power of $0 \mathrm{dBm} / \mathrm{ch}$. Each DFRA is counter-directionally pumped with two optical pumps, at $1454 \mathrm{~nm}$ and $1461 \mathrm{~nm}$, with $190 \mathrm{~mW}$ and $160 \mathrm{~mW}$, respectively. These conditions allow us to compensate for the fiber loss along the $40 \mathrm{~km}$ span.

To simulate channel removal/addition, the channel number $20(1560.2 \mathrm{~nm})$ has been used as a compensation channel, while some of the WDM channels are $100 \%$ squarewave-modulated with $500 \mathrm{~Hz}$ frequency.

\section{ClaSSICAL LINK CONTROL SCHEME}

In this case, we analyze the situation when the classical link control scheme is used [10]. For that, 9 and 18 out of 20 channels are added/dropped at the input of the first DFRA. In the first case, channels number $2,4,6,8,10,12,14,16$ and 18 are on/off modulated, while in the second case, channels number 2 to 19 are modulated. Power transients are observed from the surviving channel (Ch. 1). Fig. 2 shows the power variation of the surviving channel at the output of DFRA\#1 in both cases. We can observe that the power variation of the surviving channel reaches $0.37 \mathrm{~dB}$ when no control is applied in the first case (adding/dropping only 9 channels), while in the second case, the power transient reaches $0.7 \mathrm{~dB}$ (when adding/dropping 18 channels). When using the control channel in the system (channel 20 at $1560.2 \mathrm{~nm}$ ), transients are reduced down to $0.085 \mathrm{~dB}$ and $0.14 \mathrm{~dB}$ for the first and second cases, respectively. Even if the power transient of the surviving channels is reduced, there is still a transient which cannot be accepted at the output of the first amplifier of a link. This transient will be amplified along a cascade of DFRAs, producing an unacceptable power variation of the surviving channels at the far end of the link.

Note that Raman amplification systems depend on both the power level of the involved optical waves and the frequency difference between them. For that reason, the use of one control channel is not able to compensate for all the energy transfer occurring between pumps and signals, giving rise to the observed power transients. This situation becomes even worst for broadband transmission systems, where it is clearly

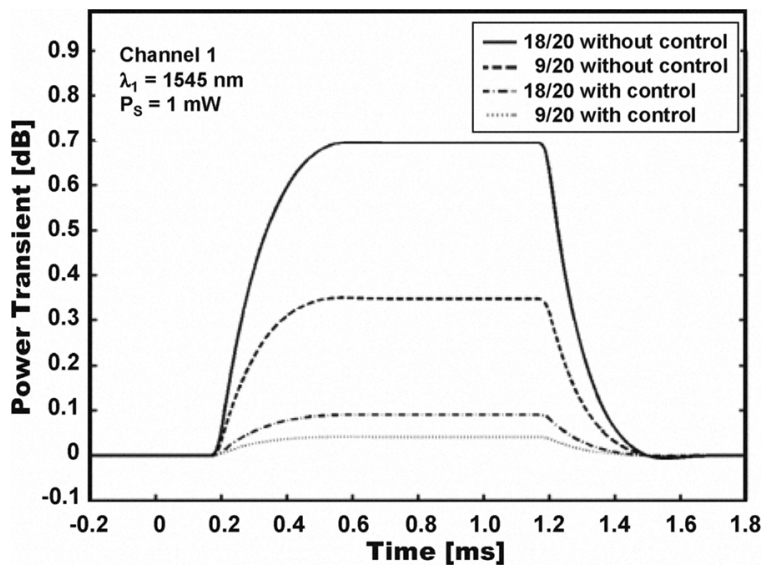

Figure 2. Power excursion of surviving channel (Ch.1) at the output of DFRA\#1, with link control on/off when 9 and 18 out of 20 WDM channels are dropped/added. evident that the use of only one control channel is not enough. In the next section we present a method for compensating power transients by using several control channels, in order to get better control performance. In particular, the description and simulation results -in next section - are shown for the case of using only 2 channels for compensation.

\section{PER-BAND LINK CONTROL SCHEME}

In Fig. 3 the use of a different link control module is proposed. This takes into account the use of two compensation channels, spectrally placed in the WDM transmission band. Thus, the first control channel $\left(\mathrm{cc}_{1}\right)$, at wavelength $\lambda_{\mathrm{cl}}$, compensates channels $c_{1}, c_{2}, \ldots c_{n}$, at wavelengths $\lambda_{1}, \lambda_{2}, \ldots, \lambda_{v}$, while the second control channel $\left(\mathrm{cc}_{2}\right)$, at wavelength $\lambda_{\mathrm{c} 2}$, compensates channels $c_{1}, c_{2}, \ldots c_{n}$, at wavelengths $\lambda_{n+1}, \ldots, \lambda_{m}$, as shown Fig. 4.

The proposed solution requires the link control module shown in Fig 3 instead of using the one shown in Fig.1. Modifications consider the inclusion of additional filters in order to separate channels in two groups and to measure their total optical power independently. Thus, the optical-bandpass filter $\mathrm{OBPF}_{1}$ selects the channels at $\lambda_{1}, \ldots, \lambda_{\mathrm{n}}$ and $\lambda_{\mathrm{c} 1 \text {, while }}$ $\mathrm{OBPF}_{2}$ selects channels at $\lambda_{\mathrm{n}+1}, \ldots, \lambda_{\mathrm{m}}$ and $\lambda_{\mathrm{c} 2}$. With this

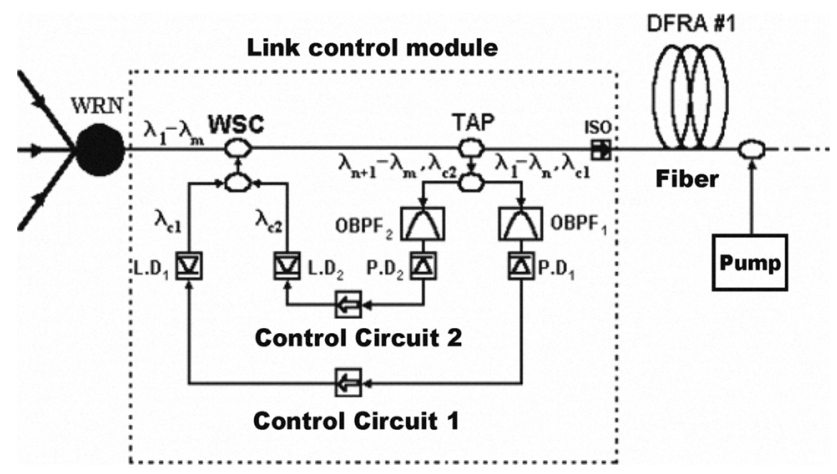

Figure 3. Schematic representation of new per-band link control module using two compensation channels $\left(\lambda_{\mathrm{c} 1}, \lambda_{\mathrm{c} 2}\right)$. Two optical band-pass filters $\left(\mathrm{OBPF}_{1,2}\right)$ detect the power variation of each signal band.

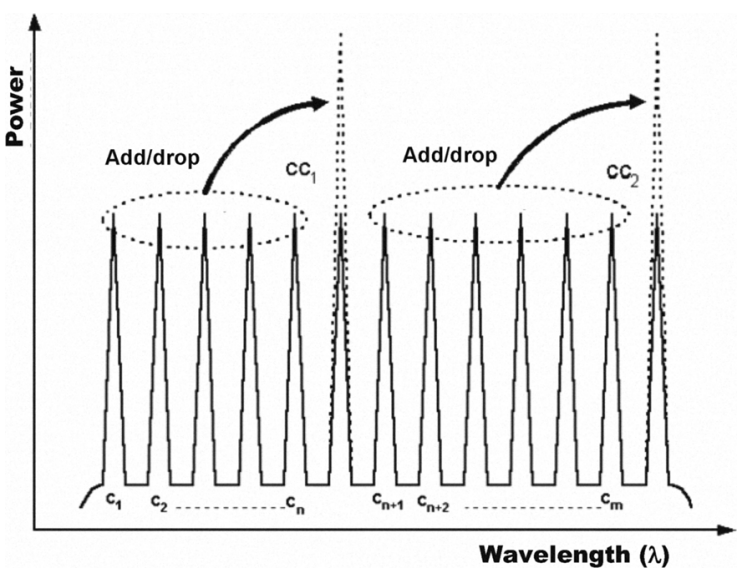

Figure 4. Spectral schematic of the per-band control channel. $\mathrm{c}_{1}, \mathrm{c}_{2}, \ldots \mathrm{c}_{\mathrm{n}}$, $\mathrm{c}_{\mathrm{n}+1}, \mathrm{c}_{\mathrm{n}+2} \ldots \mathrm{c}_{\mathrm{m}}$ : WDM channels; $\mathrm{cc}_{1}$ : compensation channel $1 ; \mathrm{cc}_{2}$ : compensation channel 2 . 
configuration, the compensation of the two groups of channels is carried out independently of each other. It is then also required to use two independent opto-electronic circuits, considering different control circuits as shown Fig. 3.

These two independent PI controllers are described by the following equations [10]:

$$
\begin{gathered}
\tau_{i} \frac{d P_{c c 1}(z=0, t)}{d t}=k_{p} R_{1}(t)+k_{p} \tau_{i} \frac{d R_{1}(t)}{d t} \\
\tau_{i} \frac{d P_{c c 2}(z=0, t)}{d t}=k_{p} R_{2}(t)+k_{p} \tau_{i} \frac{d R_{2}(t)}{d t}
\end{gathered}
$$

where $R_{l, 2}(t)$ are error functions defined by equation (2). The values of the parameters $k_{p}$ and $\tau_{i}$ are the same used for the case of a single control circuit.

In order to verify the effectiveness of the proposed solution, a simulation is carried out under the same conditions previously analyzed, but including the two control channels. Control channel 1 has been placed at 1552.2 (as Ch. 10), while control channel 2 has been placed at $1560.2 \mathrm{~nm}$ (as Ch. 20), similar to the case studied in Section V.

Fig. 5 shows the power variation of one of the surviving channels, the one at $1545 \mathrm{~nm}$ (Ch. 1), at the output of the first DFRA, with and without the new link control module. In this case, 9 and 17 out of 20 channels are dropped/added at $\mathrm{t}=0.2 \mathrm{~ms}$ and then added at $\mathrm{t}=1.2 \mathrm{~ms}$. When the new link control module is not used, the power variation of the observed channel reaches $0.33 \mathrm{~dB}$ when 9 channels (channels number 2, $4,6,8,11,13,15,17$ and 19) are added/dropped. Meanwhile, $0.69 \mathrm{~dB}$ is the power transient observed when 17 channels (number 1 to 19 and 11 to 19 ) are added/dropped. However, when including the proposed link control scheme, those variations are reduced down to $0.01 \mathrm{~dB}$ and $0.015 \mathrm{~dB}$ respectively, which are acceptable values for the output of the first amplifier of the cascade.

The effectiveness of the proposed method can actually be evaluated when analyzing the output of the cascade. Thus, Fig. 6 shows the power variation of $\mathrm{Ch} .1$ at the output of the

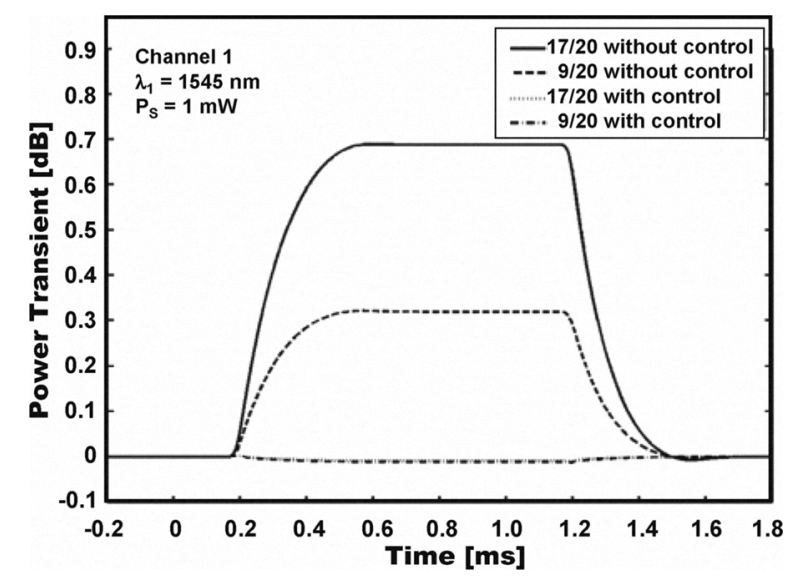

Figure 5. Power excursion of surviving channel (Ch.1, $1645 \mathrm{~nm})$ at the output of DFRA\#1, with per-band link control on/off when 9 and 17 out of $20 \mathrm{WDM}$ channels are dropped/added.

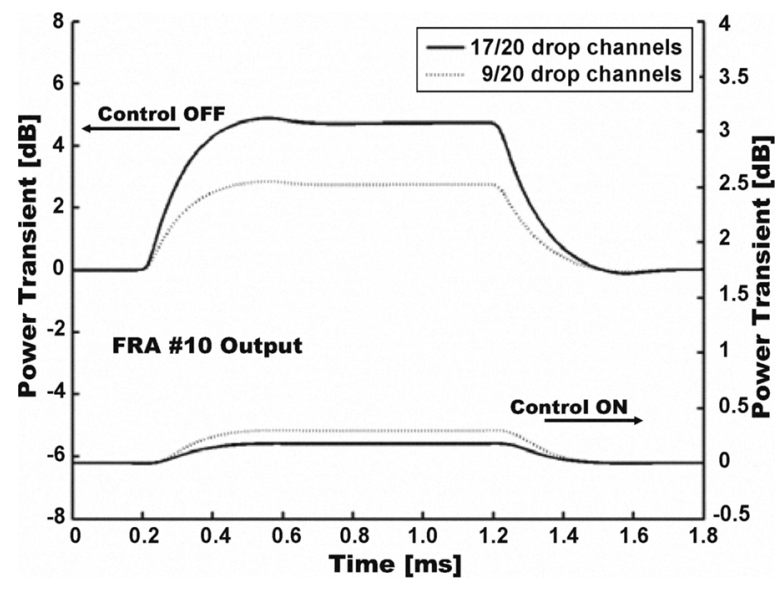

Figure 6. Power excursion of surviving channel (Ch.1, $1645 \mathrm{~nm})$ at the output of DFRA\#10, with per-band link control on/off when 9 and 17 out of $20 \mathrm{WDM}$ channels are dropped/added.

DFRA\#10 into the cascade, when 9 and 17 out of 20 channels are added/dropped.

It can be clearly seen that, when no link control is used, a transient of $2.85 \mathrm{~dB}$ occurs when 9 out of 20 channels are added/dropped, and $4.88 \mathrm{~dB}$ when 17 out of 20 channels are added/dropped. However, when using the proposed link control module, transients are reduced down to $0.24 \mathrm{~dB}$ and $0.3 \mathrm{~dB}$ respectively. Results clearly show the effectiveness of the perband link control method in Raman amplified broadband systems.

\section{CONCLUSIONS}

An innovative study of the extension of the link control technique to mitigate power transients in cascades of wideband DFRAs was presented, considering extreme conditions of channel adding/dropping. Using comprehensive and rigorous mathematical models for DFRAs and electronic control circuits, we simulated and limited the power excursion of surviving channels in a 10 DFRAs cascade placed in WRONs, using the per-band link control protection technique. Results show that the power transients of surviving channels can be drastically mitigated. In the cascade of 10 DFRAs, the power variation is reduced from $4.88 \mathrm{~dB}$ to $0.24 \mathrm{~dB}$, when 17 out of 20 channels are added or dropped. This suppression of transients is achieved by simply introducing two compensation channels at the input of the cascade in order to keep the total input power constant. This technique shows a good performance when reducing power transients, even if only two compensation channels are used, offering a potentially low-cost implementation. This feature makes this technique a highly attractive alternative for power transients suppression in broadbandwidth WRONs, where more compensation channels can be used if required.

\section{ACKNOWLEDGMENTS}

The authors wish to acknowledge the support received from the Chilean Agency CONICYT (under Fondecyt project \#1010437) and from the UTFSM project DGIP-230964. 


\section{REFERENCES}

[1] M. N. Islam, "Raman Amplifiers for Telecommunications", IEEE J. Select Topics Quantum Electron., vol. 8, no. 3, pp. 548-559, May/Jun. 2002.

[2] M. Maeda, "Operation and management of WDM optical networks", in OFC'96 Tech. Dig., San Jose, CA, (1996), FD4, 318-319.

[3] K. K. Motoshima, L. M. Leba, D. N. Chen, M. M. Downs, T. Li and E. Desurvire, "Dynamic Compensation of Transient Gain Saturation in Erbium-Doped Fiber Amplifiers by Pump Feedback Control," IEEE Photon. Tech. Lett., vol. 5, no. 12, pp. 1423-1426, 1993.

[4] J. L. Zyskind, A. K. Srivastava, Y. Sun, J. C. Ellson, G. W. Newsome, R. W. Tkach, A. R. Chraplyvy, J. W. Sulhoff, T. A. Strasser, J. R Pedrazzani, and C. Wolf, "Fast Link Control Protection for Surviving Channels in Multiwavelength Optical Networks," Proc. 22nd European Conf. on Optical Comm., ECOC'96, vol.5, pp. 49-52, 1996.

[5] R. Lebref, B. Landousies, T. Geroges and E. Delavaque, "Study of Power Transients in EDFA With Gain Stabilization by a Laser Effect," Elect. Lett., vol. 33, no. 3, pp. 191-193, 1997.

[6] Y. Sun, G. Luo, J. L. Zyskind, A. A. M. Saleh, A. K. Srivastava, and J. W. Sulhoff, "Model for Gain Dynamics in Erbium-Doped Fiber Amplifiers," Electron. Lett., vol. 32, pp. 1490-1491, 1996.
[7] S. R. Chinn, "Simplified Modeling of Transients in Gain-Clamped Erbium-Doped Fiber Amplifiers," J. Lightwave Techn., vol. 16, no. 5, pp. 1095-1100, 1998.

[8] M. Karasek and M. Menif, "Channel addition/removal response in Raman fiber amplifiers: Modeling and Experimentation", J Lightware Technol, vol. 20, pp. 1680-1687, 2002.

[9] C. J. Chen et al., "Control of transient effects in distributed and lumped Raman amplifiers", Electron Lett., vol. 37, pp. 1304-1305, 2001

[10] V. Pincheira, H. Soto and R. Olivares, "Link control protection of surviving channels in distributed fiber Raman amplifier cascades", in Proceedings of SPIE, vol. 5622, RIAO/OPTILAS 2004 5th Iberoamerican Meeting on Optics and 8th Latin American Meeting on Optics, Laser, and Their Applications, Papers 5622-62, pp. 331-335, Porlamar, Isla Margarita, Venezuela, 2004.

[11] R. W. Freund, "Simulation of Raman amplification", Bell Laboratories Murray Hill New Jersey, 2002. Available on WWW at http://cm.belllabs.com/cs/doc/02.

[12] F. Ellrich, "Measurements of the temperature dependence of the spectral Raman gain coefficient and its spectral attenuation coefficient for various songles mode Fibers", M. S. Thesis, Dept. Electron. Eng., Kaiserslautern Univ., Kaiserslautern, Germany, 2001. 